\title{
ORIGINAL ARTICLE \\ Circulating microRNAs as biomarkers for evaluating the severity of acute spinal cord injury
}

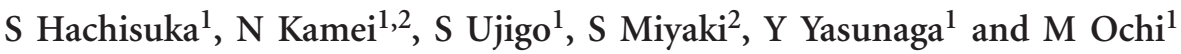

Study design: An in vivo study in mouse models of spinal cord contusion.

Objectives: To develop a novel indicator to anticipate the severity of spinal cord injury (SCI) during the acute phase and for the assessment of the efficacy of novel therapies. MicroRNAs (miRNAs) circulating in the peripheral blood are reported to modulate signaling between cells, and to be diagnostic markers for cancers. The purpose of this study was to identify circulating miRNAs for predicting the severity of $\mathrm{SCl}$ in the acute phase.

Setting: Department of Orthopaedic Surgery, Graduate School of Biomedical and Health Sciences, Hiroshima University, Hiroshima, Japan.

Methods: Mouse SCl models were made using Infinite Horizon impactor with 50 or 70 kdyn compressing power following thoracic laminectomy. The mice were then divided into four groups: normal (without surgery), sham (laminectomy only), mild (50 kdyn), and severe (70 kdyn). TaqMan low-density array analysis and real-time PCR were performed to identify candidate miRNAs that were increased in the serum relative to the severity of $\mathrm{SCl}$.

Results: The expression levels of miR-9*, miR-219 and miR-384-5p in the serum were significantly increased relative to the severity of $\mathrm{SCl} 12 \mathrm{~h}$ after injury. The expression of miR-9* was also significantly increased relative to injury severity at 3 and $24 \mathrm{~h}$ after injury. Conclusion: Serum miR-9*, miR-219 and miR-384-5p might be promising biomarkers for predicting the severity of SCl.

Spinal Cord (2014) 52, 596-600; doi:10.1038/sc.2014.86; published online 3 June 2014

\section{INTRODUCTION}

Spinal cord injury (SCI) is a serious clinical problem, because established treatments for it are currently limited. Although administration of a high dose of methylprednisolone is an established clinical therapy for SCI, it is no longer widely used due to its limited efficacy and significant side effects. ${ }^{1}$ On the other hand, there are many recent basic research studies and ongoing clinical trials concerning novel therapies for SCI. ${ }^{2}$ However, it is often difficult to determine the baseline severity of the injury due to the unstable conditions of patients, including the phenomenon of spinal shock in the acute phase. This is an obstacle to the development of new treatments for SCI during the acute phase. To assess the efficacy of novel therapies for SCI, monitoring methods for confirming the therapeutic effects are necessary. ${ }^{3}$

Based on their potential in modulating signaling not only within cells but also between cells, recent discoveries of extracellular and circulating microRNAs (miRNAs) have focused on their use as novel biomarkers. ${ }^{4}$ Profiling of circulating miRNAs has been used in a number of studies to identify novel minimally invasive biomarkers associated with cancer development and progression. ${ }^{5}$ Another important feature is that circulating miRNAs are stabilized by containing them within microvesicles that are small enough to pass through the blood-spinal cord barrier. ${ }^{6}$ In this study, we evaluated the expression of miRNAs in the serum of mice after SCI and identified candidates for novel biomarkers to evaluate the severity of SCI during the acute phase.

\begin{abstract}
MATERIALS AND METHODS
Animals

All of the experimental research protocols were reviewed and approved by the Hiroshima University ethical committee, and all experiments were performed according to an institutionally approved protocol in accordance with the National Institute of Health Guide for Care and Use of Laboratory Animals. Female C57BL/6 mice, approximately 10 weeks of age, were used.
\end{abstract}

\section{Surgical procedure}

Mice were anesthetized via sustained inhalation of $2 \%$ isoflurane in $1.51 \mathrm{~min}^{-1}$ air. Laminectomy was carried out at the tenth thoracic spinal vertebrae. A contusive SCI was induced using the Infinite Horizon impactor (Precision Systems and Instrumentation, Lexington, KY, USA). The mice were then divided into four groups: normal (without surgery), sham (laminectomy only), mild (50 kdyn compressing power), and severe (70 kdyn compressing power).

\section{Behavioral testing}

The recovery of hindlimb motor function was assessed using the Basso Mouse Scale. ${ }^{7}$ Mice in all the groups were assessed at 3,12 and $24 \mathrm{~h}$ and at 3, 5, 7, 14, $21,28,35$ and 42 days after injury ( $n=8$ in each group).

\section{Sample collection}

We collected blood samples and spinal cord tissue samples from all the four groups. Mice were killed, and blood samples $(0.6-1.0 \mathrm{ml})$ were collected from the heart at 3,12 and $24 \mathrm{~h}$ and at 3 and 7 days after injury. To harvest cell-free serum, blood was drawn into a sterile tube without anticoagulant. After

${ }^{1}$ Department of Orthopaedic Surgery, Graduate School of Biomedical and Health Sciences, Hiroshima University, Hiroshima, Japan and ${ }^{2}$ Translational Research Medical Center Hiroshima University Hospital, Hiroshima, Japan

Correspondence: Dr S Hachisuka, Division of Clinical Medical Science, Department of Orthopaedic Surgery, Graduate School of Biomedical and Health Sciences, Hiroshima University, 1-2-3 Kasumi, Minami-ku, Hiroshima 734-8551, Japan.

E-mail: s_hachi5581@yahoo.co.jp

Received 9 January 2014; revised 7 April 2014; accepted 1 May 2014; published online 3 June 2014 
standing upright at $37^{\circ} \mathrm{C}$ for $60 \mathrm{~min}$, samples were centrifuged at $2500 \mathrm{~g}$ for $30 \mathrm{~min}$ at $4{ }^{\circ} \mathrm{C}$; the supernatant serum was then removed and centrifuged again at $2900 \mathrm{~g}$ for $10 \mathrm{~min}$. The supernatant was then quickly removed and stored immediately at $-80^{\circ} \mathrm{C}$ until further testing. After harvesting the blood, mice were transcardially perfused with $10 \mathrm{ml}$ sterile RNase-free phosphate-buffered saline. Five-mm-long sections of the injured spinal cord were rapidly isolated, placed on ice and processed immediately for RNA isolation.

\section{RNA isolation}

Total RNA was isolated from $200 \mu \mathrm{l}$ of serum blood using the mirVana PARIS kit (Ambion, Foster City, CA, USA) following the manufacturer's instructions for liquid samples. Caenorhabditis elegans spiked-in oligonucleotides (cel-miR39) were introduced after denaturation for normalization of variability in RNA isolation across samples. ${ }^{8}$ Total RNA was extracted from spinal cords using the TRIzol reagent (Invitrogen, Carlsbad, CA, USA) according to the manufacturer's instructions.

\section{TaqMan low-density array for miRNA}

In the screening phase, we performed TaqMan low-density array analyses (Life Technologies, Carlsbad, CA, USA) to identify differentially expressed miRNAs in the serum samples of the normal, sham, mild and severe groups $(n=1$ in each group). A middle-throughput screening of serum miRNAs was performed using TaqMan Array Rodent MicroRNA Cards (Card A v2.0 and Card B v3.0). A spiked-in control (ath-miR159) was used as an internal control for normalization.

\section{Real-time PCR analysis}

The expression levels of miRNAs in the serum and spinal cord tissues were assessed by real-time PCR analysis ( $n=8$ in each group). Cel-miR-39 in the serum and snoRNA-202 in the spinal cord tissue were used as internal controls for normalization. The primer and TaqMan probe sequences are shown in Supplementary Table S1. The expression levels of individual miRNAs in each group were calculated relative to their mean expression level in the normal group.

\section{Statistical analysis}

Statistical analyses were performed using two-way repeated measures analysis of variance and Bonferroni post hoc comparisons for behavioral testing. The values of Basso Mouse Scale are presented as mean \pm s.e. One-way analysis of variance followed by Bonferroni multiple comparison tests were used to compare the expression levels of miRNAs in real-time PCR analyses. These values are presented as mean \pm s.d. For all analyses, statistical significance was set at $<5 \%$

\section{RESULTS}

\section{Behavioral assessment}

Sham and normal groups showed no motor function deficits (Basso Mouse Scale score 9), indicating that these groups were unaffected by the procedure, whereas all mice in the mild and severe groups were completely paralyzed $3 \mathrm{~h}$ after SCI. A significant improvement was seen in the mild group compared with the severe group beginning $12 \mathrm{~h}$ after SCI (Figure 1 ).

\section{Changes in miRNA expression levels}

To identify miRNAs whose expression levels were specifically correlated with the severity of SCI, we first selected miRNAs that had high expression levels in the following order: severe group, mild group, and sham group (Figure 2). Of these, miR-9*, miR-219 and miR-384-5p were considered to be acceptable candidates for potential diagnostic biomarkers, because there were marked differences in the expression levels of these miRNAs between the mild and severe groups, and their expression levels in the sham group were similar to those in the normal group.

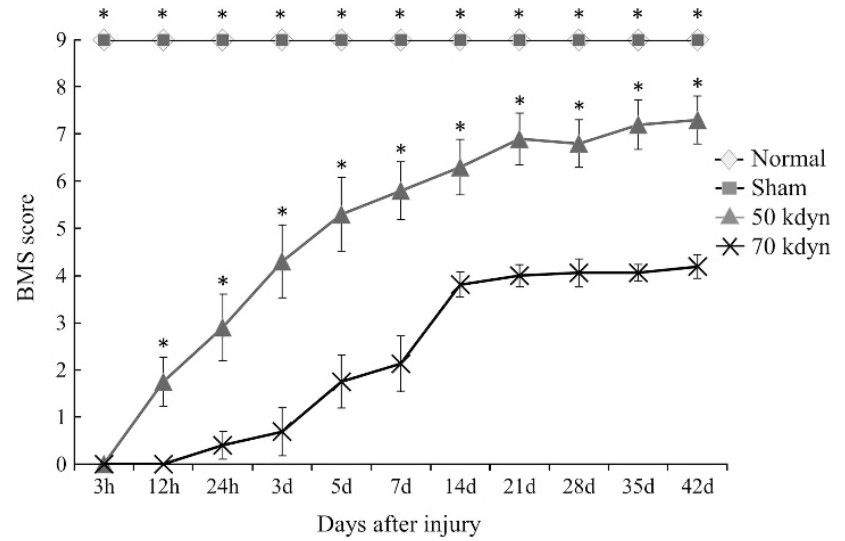

Figure 1 Assessment of functional recovery following $\mathrm{SCl}(n=8)$. A time course of functional recovery of hindlimbs assessed using the Basso Mouse Scale (BMS). The mild SCl group (50 kdyn) showed significant improvements in their BMS scores compared with the severe group (70 kdyn) beginning at $12 \mathrm{~h}$ after $\mathrm{SCl}$. All values are means \pm s.e. ( $n=8$ per group). *Significantly different from the $70 \mathrm{kdyn}, P<0.05$.

The time courses of the expression levels of miR-9*, miR-219, and miR-384-5p in the serum and spinal cord tissues were then assessed by the real-time PCR. In the serum samples (Figure 3), the expression levels of miR-9*, miR-219 and miR-384-5p in the mild and severe SCI groups were increased, whereas those in the normal and sham groups did not change over time. The levels of miR-9* and miR-384-5p expression peaked at $3 \mathrm{~h}$ after SCI and that of miR-219 peaked at $12 \mathrm{~h}$ after SCI. The expression levels of all three miRNAs were significantly increased relative to the degree of injury at $12 \mathrm{~h}$ after SCI, those of miR-9* and miR-384-5p also at $3 \mathrm{~h}$ and those of miR-9* and miR-219 also at $24 \mathrm{~h}$. On the other hand, there were no significant differences in the expression levels of miR-9*, miR-219 and miR-384-5p between the normal and sham groups at any time point.

In the spinal cord tissues (Figure 4), the expression levels of miR$9^{*}$, miR-219 and miR-384-5p in the mice with SCI peaked at $12 \mathrm{~h}$ following the injury and were significantly higher than those in the sham group at this time. However, there were no significant differences in the expression levels of these miRNAs between the mild and the severe groups at any time point.

The expression levels of miR-146a-5p and miR-155, which were reported to be closely related to inflammation, were also examined to assess the expression pattern of inflammation-related miRNAs in the serum following SCI. ${ }^{9}$ The expression levels of miR-146a-5p and miR155 in the sham, mild and severe groups were increased compared with those in the normal group at 3 and $12 \mathrm{~h}$ after SCI; however, in contrast to those of miR-9*, miR-219 and miR-384-5p, these increases were not related to the severity of SCI (Figure 5).

\section{DISCUSSION}

In the present study, we identified three serum miRNAs, miR-9*, miR-219 and miR-384-5p, as promising biomarkers for evaluating the severity of SCI in the acute phase. The expression levels of all three miRNAs were increased relative to the severity of SCI within $12 \mathrm{~h}$ after injury.

In previous studies, neurostructural proteins, including neurofilaments, neuron-specific enolase and $S 100 \beta$, were reported to be serum biomarkers for SCI. ${ }^{10-12}$ Neuron-specific enolase and S100 $\beta$ might be suitable for early diagnosis, because their expression peaked $6 \mathrm{~h}$ after 

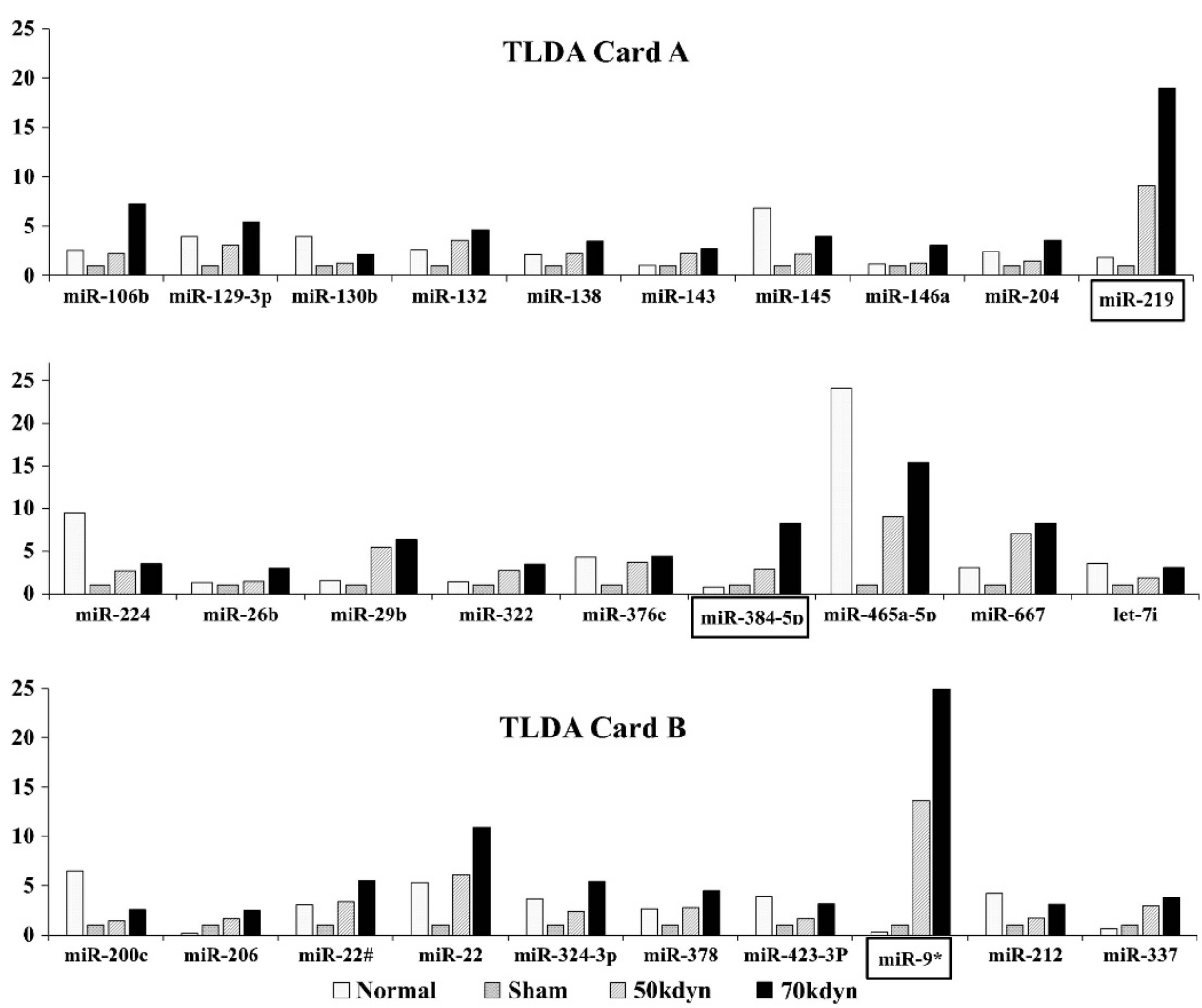

Figure 2 Results of the TaqMan low-density array analysis (TLDA) for miRNAs at $12 \mathrm{~h}$ after SCl. The miRNAs displayed showed increased expression levels relative to the severity of the SCl. MiR-9*, miR-219 and miR-384-5p were further selected from the displayed genes as candidates for biomarkers, because their expression levels in the sham group were similar to those in the normal group.
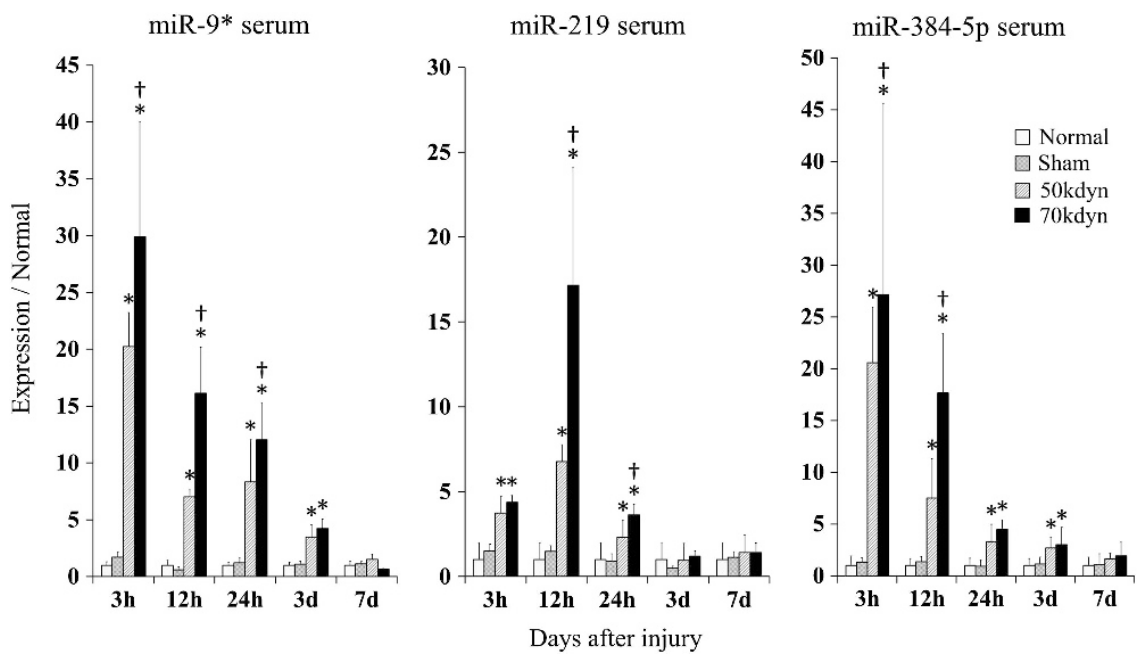

Figure 3 Real-time PCR analysis of the expression levels of miR-9*, miR-219 and miR-384-5p in serum samples from mice in the normal, sham, mild $(50 \mathrm{kdyn})$ and severe $(70 \mathrm{kdyn})$ groups over time following the SCl. All values are means $\pm \mathrm{s} . \mathrm{d}$. ( $\mathrm{n}=8$ per group). ${ }^{*}$ Significantly greater than the sham groups, `significantly greater than the $50 \mathrm{kdyn}$ group, $P<0.05$.

SCI. However, the sensitivity of these proteins to predict the severity of SCI varied by reports, and one of them did not show a significant difference relative to the severity of SCI. ${ }^{11}$ On the other hand, the phosphorylated form of the high-molecular-weight neurofilament subunit (pNF-H) in serum was recently reported to be a promising biomarker for evaluating the severity of SCI. Serum pNF-H was reported to increase in patients with SCI. The serum pNF-H values of patients with complete SCI, classified as American Spinal Injury Association impairment scale (AIS) A, were markedly higher than those of patients with incomplete SCI (AIS C, D and E). However, there was no significant difference in serum pNF-H levels between AIS C and D patients. Therefore, pNF-H can be applied only to 

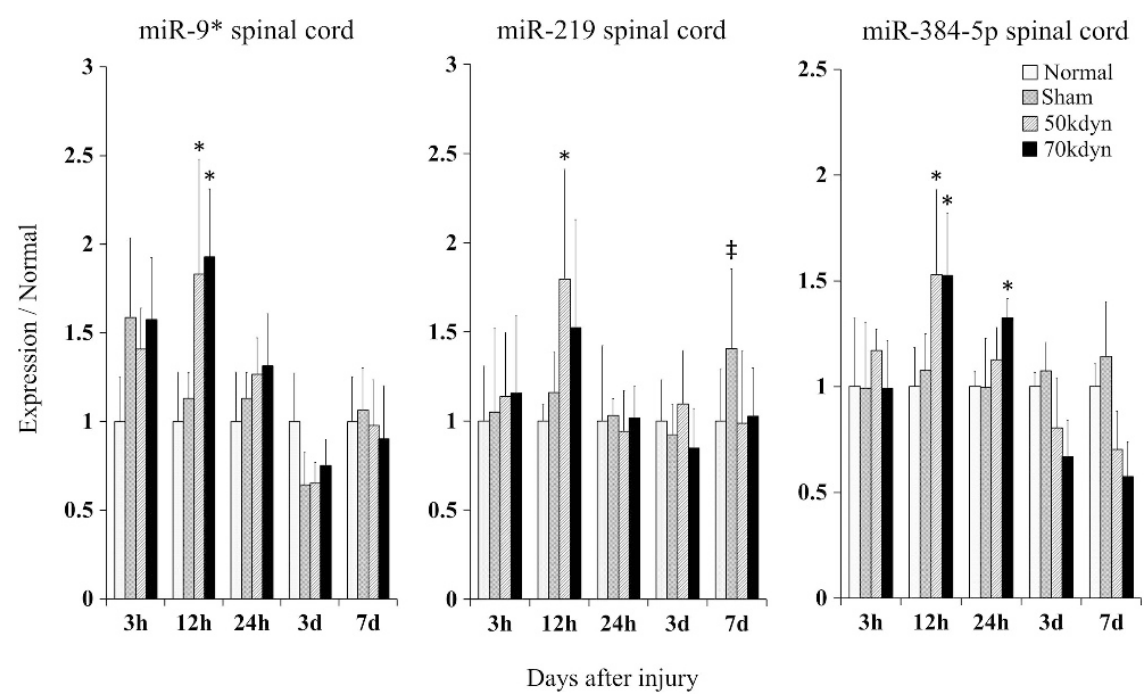

Figure 4 Real-time PCR analysis of the expression of miR-9*, miR-219 and miR-384-5p in spinal cord tissues. All values are means $\pm s . d$. ( $n=8$ per group). *Significantly greater than the sham group, ${ }^{\ddagger}$ significantly greater than the $70 \mathrm{kdyn}$ group, $P<0.05$.

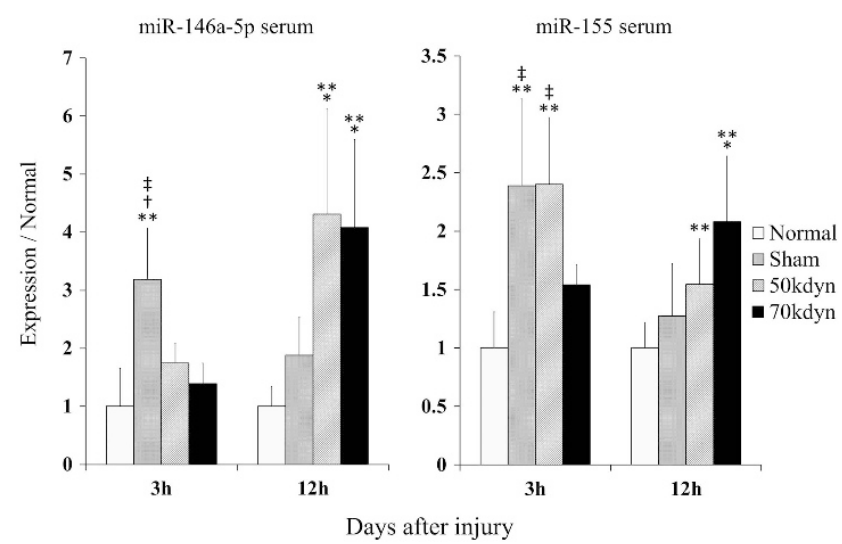

Figure 5 Real-time PCR analysis of the expression in serum of miR-146a$5 \mathrm{p}$ and miR-155, both of which were reported to have a close relation to inflammation. All values are means \pm s.d. ( $n=8$ per group). *Significantly greater than the sham group, ${ }^{*}$ significantly greater than the normal group, †significantly greater than the $50 \mathrm{kdyn}$ group, $\ddagger$ significantly greater than the 70 kdyn group, $P<0.05$.

distinguish complete SCI from incomplete SCI. Additionally, it takes $>18 \mathrm{~h}$ to determine the severity of SCI using serum pNF-H, ${ }^{10}$ and acute-phase treatments for SCI might require them to be started earlier. For example, methylprednisolone needs to be administrated within $8 \mathrm{~h}$ of SCI. ${ }^{13}$ It is therefore desirable to identify novel sensitive biomarkers that enable earlier diagnosis of the severity of SCI. MiRNAs are thought to have high value as biomarkers due to the highly sensitive PCR detection methods and their low complexity compared with protein biomarkers. ${ }^{14}$ In the current study, the expression levels of miR-9*, miR-219 and miR-384-5p all detected a difference in the severity of SCI at $12 \mathrm{~h}$ after SCI. Additionally, the expression of miR-9* was also increased relative to SCI severity at 3 and $24 \mathrm{~h}$ after SCI. Therefore, these miRNAs might enable us to predict the severity of SCI earlier than pNF-H. However, it is unclear whether the kinetics of these miRNAs are similar in mice and humans. The expression levels of these miRNAs in clinical serum samples should be examined in the next step.
Previously, Redell et al. ${ }^{15}$ reported that plasma levels of miR-92a and miR-16 in severe traumatic brain injury were significantly increased within the first $24 \mathrm{~h}$ compared with healthy volunteers. However, the result of TaqMan low-density array in our present study showed negative association between the expression level of miR-92a and miR-16 and the severity of SCI. On the other hand, Liu et al. ${ }^{16}$ performed miRNA microarray assay using spinal cord tissues of SD rats and described several miRNAs as targets for therapeutic interventions. Our previous study also reported a marked change of expression levels of mir-124a and mir-223 in spinal cord tissue of mice following SCI. ${ }^{17}$ However, the expression levels of serum miRNAs were not reported in these previous reports. Additionally, the expression levels of these miRNAs in the serum were not specifically associated with the severity of SCI in miRNA microarray assay in the present study. Therefore, the present study is the first report of the serum miRNAs as biomarkers for the severity of SCI.

We cannot say whether serum miR-9*, miR-219 and miR-384-5p were derived from the injured spinal cord. At least, the increases in these miRNAs in the serum did not reflect their upregulation in spinal cord tissue. Because the levels of total RNA in the spinal cord tissues were higher than that in the serum, the absolute amount of miRNA, including miR-9*, miR-219 and miR-384-5p, in the spinal cord tissues must be much larger than that in the serum. This large difference in the absolute amount of miRNA between spinal cord tissues and serum might be related to the discrepancy in the expression levels of miR-9*, miR-219 and miR-384-5p between spinal cord tissues and serum (for example, minimal changes in the expression levels of these miRNAs in spinal cord tissues might have large effects on their expression levels in serum). However, previous studies showed that miR-9 and miR-219 were detected in oligodendrocyte lineage cells and had an important role in oligodendrocyte differentiation and myelin maintenance. ${ }^{18-20}$ Therefore, increases in serum miR- $9^{*}$ and miR-219 might be associated with the release of these miRNAs into the circulation resulting from the destruction of myelin in the spinal cord. On the other hand, it is difficult to discuss the origin of miR384-5p, because little is known about its tissue-specific expression or function. In the present study, the expression levels of these serum miRNAs were unaffected by the skeletal muscle and bone injuries associated with laminectomy. Furthermore, the expression patterns of 
these serum miRNAs were quite different from those of inflammation-related miRNAs in the serum. These findings suggest that increases in these miRNAs were at least specifically linked to the severity of SCI. These miRNAs may also be useful as a diagnostic tool in the patient with acute SCI who is unconscious or who cannot be immediately examined neurologically. Although further studies in clinical trials are needed to provide credible evidence, miR-9*, miR-219 and miR-384-5p are promising candidates for biomarkers of SCI.

\section{DATA ARCHIVING}

There were no data to deposit.

\section{CONFLICT OF INTEREST}

The authors declare no conflict of interest.

\section{ACKNOWLEDGEMENTS}

This work was supported by a grant-in-aid for scientific research from the Japan Orthopaedics and Traumatology Foundation. TaqMan low-density array analyses in this study were carried out at the Analysis Center of Life Science, Natural Science Center for Basic Research and Development, Hiroshima University, Hiroshima, Japan.

1 Bracken MB, Shepard MJ, Holford TR, Leo-Summers L, Aldrich EF, Fazl M et al. Administration of methylprednisolone for 24 or 48 hours or tirilazad mesylate for 48 hours in the treatment of acute spinal cord injury. Results of the third national acute spinal cord injury randomized controlled trial. National Acute Spinal Cord Injury Study. JAMA 1997; 277: 1597-1604

2 Silva NA, Sousa N, Reis RL, Salgado AJ. From basics to clinical: a comprehensive review on spinal cord injury. Prog Neurobiol 2014; 114: 25-57.

3 Yokobori S, Zhang Z, Moghieb A, Mondello S, Gajavelli S, Dietrich WD et al. Acute diagnostic biomarkers for spinal cord injury: review of the literature and preliminary research report. World Neurosurg (e-pub ahead of print 19 March 2013; doi:10.1016/ j.wneu.2013.03.012)
4 Chen X, Liang H, Zhang J, Zen K, Zhang CY. Secreted microRNAs: a new form of intercellular communication. Trends Cell Biol 2012; 22: 125-132.

$5 \mathrm{Mo} \mathrm{MH}$, Chen L, Fu Y, Wang W, Fu SW. Cell-free circulating miRNA biomarkers in cancer. J Cancer 2012; 3: 432-448.

6 Valadi H, Ekström K, Bossios A, Sjöstrand M, Lee JJ, Lötvall JO. Exosome-mediated transfer of mRNAs and microRNAs is a novel mechanism of genetic exchange between cells. Nat Cell Biol 2007; 9: 654-659.

7 Basso DM, Fisher LC, Anderson AJ, Jakeman LB, McTigue DM, Popovich PG. Basso Mouse Scale for locomotion detects differences in recovery after spinal cord injury in five common mouse strains. J Neurotrauma 2006; 23: 635-659.

8 Mitchell PS, Parkin RK, Kroh EM, Fritz BR, Wyman SK, Pogosova-Agadjanyan EL et al. Circulating microRNAs as stable blood-based markers for cancer detection. PNAS 2008; 105: 10513-10518.

9 Roy S, Sen CK. MiRNA in innate immune responses: novel players in wound inflammation. Physiol Genomics 2011; 43: 557-565.

10 Hayakawa K, Okazaki R, Ishii K, Ueno T, Izawa N, Tanaka Y et al. Phosphorylated neurofilament subunit NF-H as a biomarker for evaluating the severity of spinal cord injury patients, a pilot study. Spinal Cord 2012; 50: 493-496

11 Loy DN, Sroufe AE, Pelt JL, Burke DA, Cao QL, Talbott JF et al. Serum biomarkers for experimental acute spinal cord injury: rapid levation of neuron-specific enolase and S-100beta. Neurosurgery 2005; 56: 391-397.

12 Ma J, Novikov LN, Karlsson K, Kellerth JO, Wiberg M. Plexus avulsion and spinal cord injury increase the serum concentration of S-100 protein: an experimental study in rats. Scand J Plast Reconstr Surg Hand Surg 2001; 35: 355-3591.

13 Bracken MB, Shepard MJ, Collins WF, Holford TR, Young W, Baskin DS et al. A randomized, controlled trial of methylprednisolone or naloxone in the treatment of acute spinal cord injury. New Engl J Med 1990; 322: 1405-1411.

14 Wittmann J, Jack HM. Serum microRNAs as powerful cancer biomarkers. Biochim Biophys Acta 2010; 1806: 200-207.

15 Redell JB, Moore AN, Ward NH 3rd, Hergenroeder GW, Dash PK. Human traumatic brain injury alters plasma microRNA levels. J Neurotrauma 2010; 27: 2147-2156.

16 Liu NK, Wang XF, Lu QB, Xu XM. Altered microRNA expression following traumatic spinal cord injury. Exp Neurol 2009; 219: 424-429.

17 Nakanishi K, Nakasa T, Tanaka N, Ishikawa M, Yamada K, Yamasaki K et al. Responses of microRNAs 124a and 223 following spinal cord injury in mice. Spinal Cord 2010; 48: $192-196$

18 Li JS, Yao ZX. MicroRNAs: novel regulators of oligodendrocyte differentiation and potential therapeutic targets in demyelination-related diseases. Mol Neurobiol 2012; 45: 200-212

19 Lau P, Verrier JD, Nielsen JA, Johnson KR, Notterpek L, Hudson LD. Identification of dynamically regulated microRNA and mRNA networks in developing oligodendrocytes. J Neurosci 2008; 28: 11720-11730.

20 Shin D, Shin JY, McManus MT, Ptácek LJ, Fu YH. Dicer ablation in oligodendrocytes provokes neuronal impairment in mice. Ann Neurol 2009; 66: 843-857.

Supplementary Information accompanies this paper on the Spinal Cord website (http://www.nature.com/sc) 